\title{
ACCURACY OF TOOTH GUIDED IMMEDIATE IMPLANT PLACEMENT AT MOLAR REGION (CLINICAL TRIAL)
}

\author{
Abdelrhman T. Sahry ${ }^{1 *} B D S$, Samraa A. Elsheikh² $P h D$, Hala R. Ragab ${ }^{3} P h D$.
}

\begin{abstract}
INTRODUCTION: The concept of immediate implant placement has become popular due to reduced trauma and reduced overall treatment time. Removal of molar teeth provides a challenging and intriguing dilemma due to multiple root morphology. In cases of extraction and immediate placement of implants in the molar region, removal of the inter-radicular septum should be avoided to attain initial implant stability at the time of placement, and prevent slipping of the implant into the extraction socket. Following this technique prevents the slippage of the implant and allows the implant to be guided into the inter-radicular septum.

OBJECTIVES: To assess the accuracy of implant placement using the remaining roots of multi-radicular mandibular molars, evaluate bone density around implants and implant stability in fresh extracted site.

MATERIALS AND METHODS: A prospective study was done on 10 patients with badly destructed mandibular molars. The technique of implant insertion consisted of a progressive preparation of the implant site using the anatomy of the root of the multi-radicular mandibular molars to be extracted as a reference and as an aid to engage the inter-radicular septum. Implant stability was evaluated using Osstell; Radiographic evaluation was done by Cone Beam Computed Tomography.

RESULTS: Nine out of ten implants were functioning successfully along the evaluation period, while one implant failed 4 weeks after insertion and was removed. Clinically, implants stability was assessed using Osstell. All implants showed increase in ISQ value after 3 months. Radiographically, immediate postoperative CBCT was carried out which showed implants placed in ideal position. After 6 months, CBCT showed increase in bone density around implants compared to the immediate postoperative CBCT.

CONCLUSIONS: Tooth guided immediate implant placement at molar region is a novel technique for easy and safe implant insertion.

KEYWORDS: Guided surgery, immediate placement, implants bed preparation.
\end{abstract}

RUNNING TITLE: Tooth guided immediate implant placement at molar region.

1 Bachelor of Dental Surgery, Faculty of Dentistry, Alexandria University, Alexandria, Egypt.

2 Professor of Oral and Maxillofacial Surgery Alexandria University, Alexandria, Egypt.

3Assistant Professor of Oral and Maxillofacial Surgery Department, Faculty of Dentistry, Alexandria University, Alexandria, Egypt.

* Corresponding Author:

E-mail: abdosahry@gmail.com

\section{INTRODUCTION}

Immediate implant placement in fresh extraction sockets was initially described more than 30 years ago by Schulte in 1976 (1). The high success rates of this approach have been reviewed and documented demonstrating that it is a beneficial and predictable procedure for the replacement of hopeless teeth $(2,3)$.

Immediate placement of implants at the time of teeth extraction reduces the number of surgical procedures and treatment time. Moreover, it ensures an ideal three dimensional implant position, and preserves alveolar bone at the site of tooth extraction and soft tissue aesthetics and has similar survival rates when compared with the delayed protocol $(4,5)$. Commonly, immediate implants are reserved for single-root anterior teeth and single or bi-root premolar teeth. In the case of immediate placement of molar teeth, there are many difficulties regarding occlusion, site anatomy, and biomechanical issues (6).

A surgical technique that involves implant placement into the inter-radicular bone of a multi-rooted molar tooth extraction socket should be considered to take advantage of the anatomy of the trunk of the roots to guide the drilling process into the inter-radicular bone of the socket. This will aid in obtaining adequate primary stability of the implants in an ideal position regardless of the morphology of the extraction socket (7).

The tooth guided site preparation technique is a very convenient and easy technique to aid implant placement in the regions of multi-radicular teeth. This technique of implant placement comprises a progressive drilling of the implant site using the anatomy and geometry of the roots of the multiradicular teeth to be replaced as a reference and as an aid to engage the inter-radicular bony septum. This places the implants in a favorable and good position from an occlusal and biomechanical standpoint (7).

The main objective of this study is to describe a tooth-guided implant placement technique as an aid to place dental implants in multi-radicular mandibular molar teeth post-extraction sockets in a favorable position to attain predictable and favorable outcomes. The null hypothesis assumes that no effects will be found using the tooth as an anatomical guide for immediate implant placement compared to the conventional method. 


\section{MATERIALS AND METHODS}

Appropriate ethical clearance was obtained from the Faculty of Dentistry, Alexandria University and an informed consent was obtained from the participant patients. This manuscript was registered in www.clinicaltrials.gov clinicaltrials,gov identifier: NCT04192461

\section{Patients}

This study was a prospective clinical randomized controlled trial conducted on ten patients with badly destructed mandibular molars indicated for immediate implant placement who met the inclusion criteria. Patients were selected from the outpatient clinics of Oral and Maxillofacial Surgery Department Faculty of Dentistry Alexandria University.

Inclusion criteria

Medically fit patients of age ranging from 20-45 years with badly destructed mandibular molar teeth, bone coverage of $2 / 3$ of the root and sufficient bone volume.

Exclusion criteria

Patients with systemic conditions known to affect the periodontal status and healing such as uncontrolled diabetes and osteoporosis, heavy smokers, Unfavorable position of the tooth or remaining roots, molars with Fused roots, root ankylosis.

\section{Materials}

Implants system

The Dentis system implants (One Q S-clean tapered Dentis system, Woram-Dong, Dalseo-Gu, Daegu, Korea) with different diameters (3.4, 4.1 and $4.8 \mathrm{~mm}$ ) and lengths (10, 12 and $14 \mathrm{~mm}$ )

Osstell ${ }^{\mathrm{TM}}$ device

The Osstell ${ }^{\mathrm{TM}}$ (Osstell ABStampgatn 14-SE 41101 Göteborg, Sweden - www.osstell.com.) resonance frequency analyzer (RFA) is a device that uses resonance frequency analysis to measure implant stability quotient (ISQ).

\section{Methods}

Presurgical phase

Clinical examination

Evaluation of the integrity of roots, interocclusal distance and buccolingual width. (Figure $1 \mathrm{~A}$ )

Radiographic examination

Cone Beam Computer Tomography (CBCT) (PaX-i3D Green, Vatech, USA) before implant placement for assessment of the anatomy of inter-radicular septum and tooth roots, periapical pathology, bone density and approximation of inferior alveolar canal (Figure $1 \mathrm{~B}$ )

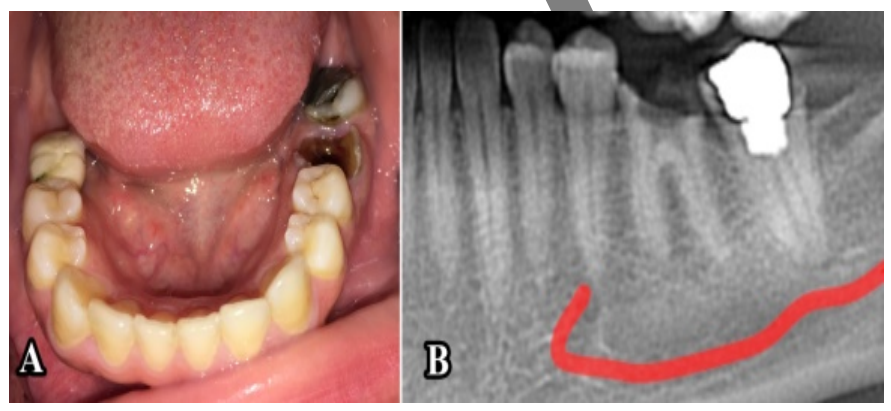

Figure (1): Preoperative view (A); photograph showing badly destructed left mandibular first molar (B): Preoperative CBCT

\section{Surgical phase}

All patients underwent the surgery under local anesthesia. Articaine hydrochloride 4\% and levonordefrin (Septanest; Septodont) were used. Coronectomy was done to the level of the free gingiva. Separation of the roots with surgical fissure bur, then drilling the implant site was done with roots intact in their sockets. A periapical $x$-ray with the final drill in the osteotomy site was done to check the proper position of the osteotomy between the roots. Clinically visible remaining roots were extracted from the socket using periotomes. Following extraction of the remaining portions of root, the socket was debrided to remove any remnants related to the process, and then the socket and the osteotomy were thoroughly irrigated with sterile normal saline to remove any debris that may be left in the site. The implant was then inserted into the site following the manufacturer's protocol. Finally, primary stability was measured using Osstell. (Figure 2)

Postsurgical phase

Postoperative instructions were given to the patients including oral hygiene instructions. All patients received postoperative medications including Amoxicillin clavulanate $1 \mathrm{gm}$ (Augmentin: manufactured by GalaxosmithKline, England); 1 capsule every 12 hours for 5 days. In addition to, Non-steroidal anti-inflammatory drugs ibuprofen $400 \mathrm{mg}$ (BRUFEN: manufactured by Abbotl), one tablet three times daily after meals for 4 days.

Patients were advised to use chlorhexidine HCL $0.12 \%$ (Hexitol: manufactured by the Arab Drug Company, Cairo, A.R) oral rinse 3 times daily for 1 week.

Clinical evaluation

Implant stability quotient (ISQ) was measured immediately and 3 months postoperative by using Osstell.

Radiographic evaluation

Immediate and 6 months post-operative cone beam computed tomography (CBCT) were done to evaluate the actual implant position and bone density around implants.

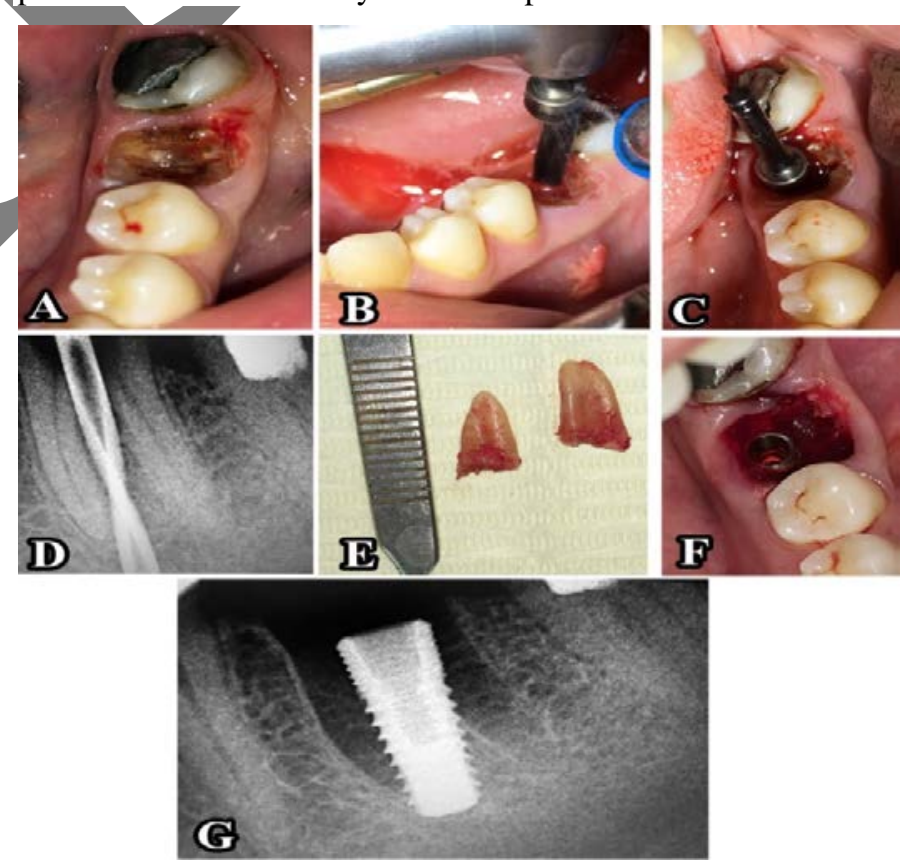

Figure (2): Tooth guided immediate implant placement technique (A): a photograph after coronectomy of the tooth (B): a photograph showing sequential drilling between roots 
(C): photograph of the implant drill for verification of the osteotomy position.(D): Periapical x-ray verifying correct osteotomy position (E):photograph showing extraction of separated roots $(\mathrm{F})$ : photograph showing implant placed in ideal position in the interradicular bone $(\mathrm{G})$ : Periapical x-ray ensuring the implant placed in the correct position

\section{Evaluation of accuracy}

Immediate postoperative CBCT scan was conducted with the same apparatus and settings as the preoperative scan.

The preoperative and postoperative scans were then superimposed, to compare the virtually planned and the actual implant positions. Three deviation parameters between each planned and placed implant were measured. All measurements were performed using Mimics Innovation Suite 19 TM software (Materialise Mimics software 3D Medical Imaging, Belgium). For analyzing the accuracy, the planned position of the implant was compared with the actual position of the implant after insertion. Several measuring points were used for the comparison of these positions:

- Horizontal deviation at coronal side of the implant.

- Horizontal deviation at apex of the implant.

- Angular deviation.

Prosthetic Protocol

Loading of the prosthesis was done 3 months postoperatively. (Figure 3)
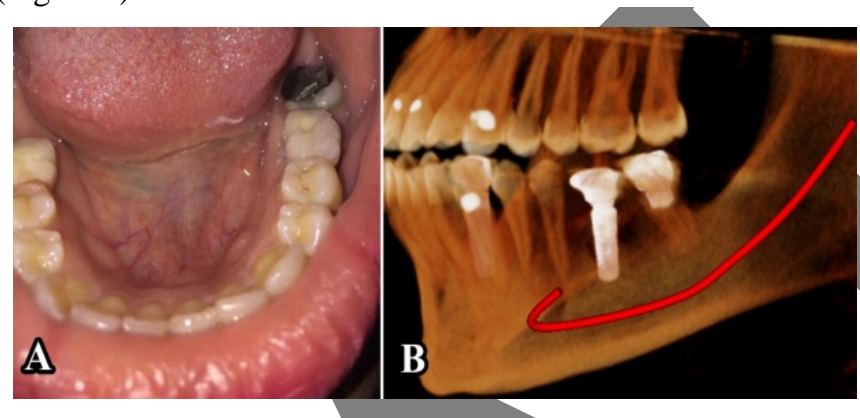

Figure (3): Postoperative view (A): Photograph showing final restoration (B): 6 months Postoperative CBCT with final restoration.

\section{Statistical analysis}

Data were fed to the computer and analyzed using IBM SPSS software (Package version 20.0. IBM Corporation, 1 New Orchard Road, Armonk, New York, United States). Quantitative data were described using range (minimum and maximum), mean, standard deviation and median. Qualitative data were described using number and percent. Paired Samples T-Test was used to analyze the significance between the different stages. To compare between the different periods Related Samples Wilcoxon Signed Rank Test was applied. Significance of the obtained results was judged at the 5\% level.

\section{RESULTS}

The present study was conducted on ten patients. Ten implants were placed in fresh extracted mandibular molars socket by using the tooth roots guiding the osteotomy. Patients were selected from the outpatient clinic of the Oral and Maxillofacial Surgery Department, Faculty of dentistry, Alexandria University. All patients were free from any systemic disease that can compromise implant success. The selected patients' ages ranged from 20-45 years old with mean of 32.7 years old and were 6 females and 4 males.
In this study, nine out of ten implants were functioning successfully along the evaluation period, while one implant failed 4 weeks after placement and was removed. This failure may be caused by the patient being non-compliant with the post-surgery instructions for good oral hygiene and not completing the medication course. Data recorded from this patient were excluded from further analysis in this study due to early implant failure; however, follow up of the patient for 12 months was done for other treatment options.

\section{Clinical evaluation}

Implant stability measured by OsstellTM Immediately and after 3 months

Results from resonance frequency analysis were based on repeated measurements per implant at 0 and 3 months on two surfaces. The measurement was done from buccal to lingual and from mesial to distal; the median readings between the two readings were recorded.

As it is shown in (Table 1), at day 0 , the measurements revealed very good stability in all implants. The implant stability quotient (ISQ) ranges of readings were between (35.0-81.0). The median records were $(55.0(40.0-68.0))$ and the Mean \pm SD (55.89 \pm 15.53$)$.

After 3 months, the measurements were taken for all the implants. All implants showed increase in ISQ value ranging between (43.0-86.0). The median records were (74.0(45.579.0)) and the Mean \pm SD (66.33 \pm 17.03$)$. (Table 1)

All implants showed an increase in ISQ value from 0 to 3 months, except for one implant which failed and was removed one month after placement.

Table (1): Comparison between the two studied periods according to stability.

\begin{tabular}{|l|c|c|c|c||}
\hline Stability (ISQ) & $\begin{array}{c}\text { Immediately } \\
(\mathrm{n}=9)\end{array}$ & $\begin{array}{c}3 \text { months } \\
(\mathrm{n}=9)\end{array}$ & $\mathrm{t}$ & $\mathrm{P}$ \\
\hline Min. - Max. & $35.0-81.0$ & $43.0-86.0$ & & \\
Mean \pm SD. & $55.89 \pm 15.53$ & $66.33 \pm 17.03$ & $4.486^{*}$ & $0.002^{*}$ \\
Median (IQR) & $55.0(40.0-68.0)$ & $74.0(45.5-79.0)$ & & \\
\hline
\end{tabular}
t:Paired t-test
p: p value for comparing between the studied periods
*: Statistically significant at $\mathrm{p} \leq 0.05$

\section{Radiographic evaluation}

Accuracy of immediate implant placement

For all ten implants, angular deviation, coronal deviation and apical deviation were determined. Data collected were statistically analyzed. (Table 2, Figure 4)

Evaluation of angular deviation

Angular deviations between the long axis of the placed implants to the virtual implants had a range of $(0.51-9.23)$ degrees with an average of $(3.78 \pm 3.22)$ degrees.

Evaluation of coronal deviation

Results show that implants placed with our technique had a range of horizontal deviation at the coronal side of the implant of $(0.20-1.90) \mathrm{mm}$ with an average of $(0.99 \pm 0.51) \mathrm{mm}$. 
Table (2): Linear (in mm) and angular (in degrees) deviations between placed and virtual implants units $(\mathrm{n}=10)$

\begin{tabular}{||l|c|c|c||}
\hline Accuracy & $\begin{array}{c}\text { Coronal } \\
\text { (mm) }\end{array}$ & $\begin{array}{c}\text { Apical } \\
\text { (mm) }\end{array}$ & $\begin{array}{c}\text { Angular } \\
\text { (degree) }\end{array}$ \\
\hline 1 & 0.81 & 1.4 & 7.93 \\
2 & 1.09 & 1.55 & 4.99 \\
3 & 1.9 & 2.3 & 9.23 \\
4 & 0.81 & 1.7 & 6.52 \\
5 & 1.46 & 1.42 & 0.64 \\
6 & 1.2 & 0.7 & 0.51 \\
7 & 1.35 & 1.16 & 0.82 \\
8 & 0.2 & 0.7 & 1.2 \\
9 & 0.57 & 0.82 & 3.2 \\
10 & 0.52 & 1.08 & 2.8 \\
\hline Min. - Max. & $0.20-1.90$ & $0.70-2.30$ & $0.51-9.23$ \\
Mean \pm SD. & $0.99 \pm 0.51$ & $1.28 \pm 0.50$ & $3.78 \pm 3.22$ \\
Median (IQR) & $0.95(0.56-1.4)$ & $1.28(0.79-1.6)$ & $3.0(0.78-6.9)$ \\
\hline
\end{tabular}
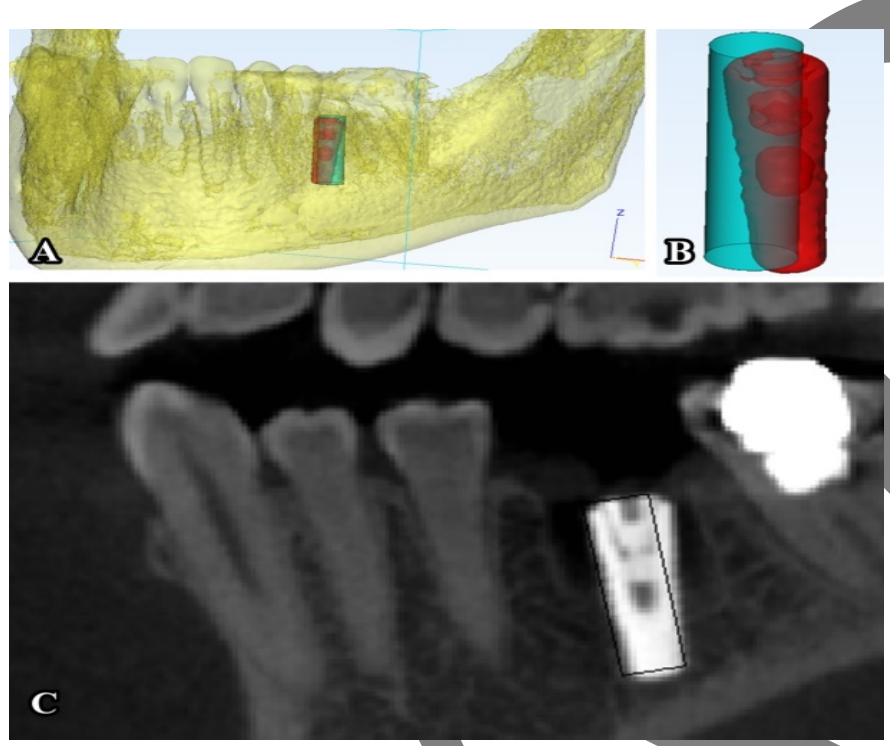

Figure (4): Evaluation of accuracy of implant placement $(A, B)$ : 3D view of a CBCT showing the actual implant in red and the virtual one in blue (C): Postoperative Sagittal view of a CBCT showing the actual implant and the virtual one (black).

\section{Evaluation of apical deviation}

Horizontal deviations at the apex of the implants had a range of $(0.70-2.30) \mathrm{mm}$ with an average of $(1.28 \pm 0.50) \mathrm{mm}$.

Assessment of bone density around the implants

Using cone beam computed tomography (CBCT) software "On Demand 3D App” immediately and after 6 months of implant placement to evaluate bone density around the implant.

Data were collected regarding mean peri-implant bone density values and standard deviation at 0 and 6 months are shown in (Table 3).

Immediately postoperative, the mean peri-implant bone density value was $685.2 \pm 279.1 \mathrm{HU}$ with a minimum recorded value of 434.9 HU and a maximum recorded value of $1193.7 \mathrm{HU}$.

At 6 months postoperative, the mean peri-implant bone density value was $780.8 \pm 281.8$ with a minimum recorded value of 491.3 HU and a maximum recorded value of $1302.7 \mathrm{HU}$
These increases in the bone density for the all implants were clear after 6 months, except for one implant which failed and was removed after 1 month. The differences between bone density immediately post-operatively and 6 months postoperatively were statistically significant $(p<0.05)$.

Post-operative complications

No post-operative complications were recorded regarding infection in the early follow up period except for one implant. The patient complained of pain and pus discharge intraorally at the site of the implant 3 weeks postoperatively, medications were prescribed to the patient for one week with no improvement, so the implant was removed after 1 month.

Table (3): Comparison between the two studied periods according to bone density

\begin{tabular}{||c|c|c|c|c||}
\hline $\begin{array}{c}\text { Bone } \\
\text { density }\end{array}$ & $\begin{array}{c}\text { Immediately } \\
(\mathrm{n}=9)\end{array}$ & $\begin{array}{c}6 \text { months } \\
(\mathrm{n}=9)\end{array}$ & $\mathrm{Z}$ & $\mathrm{P}$ \\
\hline $\begin{array}{c}\text { Min. }- \\
\text { Max. } \\
\text { Mean } \pm \\
\text { SD. } \\
\text { Median } \\
(\text { IQR })\end{array}$ & $434.9-1193.7$ & $491.3-1302.7$ & & \\
\hline
\end{tabular}

Z: Wilcoxon signed ranks test

$\mathrm{p}$ : $\mathrm{p}$ value for comparing between the studied periods

*: Statistically significant at $\mathrm{p} \leq 0.05$

\section{DISCUSSION}

The immediate dental implant placement is a widely accepted procedure, achieving survival rates comparable to the delayed implants protocol $(6,8)$. Although there are standardized protocols and numerous studies describing this technique in the esthetic zone, there is less information about the placement of immediate implants in the posterior region where the esthetical impact is lower, but the surgical difficulty can be more challenging. For example, anatomical challenges, such as differences between the size of the implant and the alveolus postextraction, root length, height of root trunk, and divergence of roots make this surgical technique more challenging $(9,10)$.

Tooth guided immediate implant placement technique is a novel technique that allowed for improved guidance during implant bed preparation for immediate implants at multi-rooted molar sites.

This technique helps in better stabilization and guidance of osteotomy drills supported by retained roots and allowed for precise three dimensional positioning of implant using maximum inter-radicular bone septa support at multi-rooted extraction sites.

In this study, nine out of ten implants were functioning successfully along the evaluation period, giving a survival rate of $90 \%$, while one implant failed 4 weeks after insertion and required removal of the implant. This failure may be due to the patient not complying with the post-surgery instructions for good oral hygiene and not completing the medication course. This was in agreement with Tolstunov in (2006) (11) who stated that poor oral hygiene is one of the main causes of early implant failure.

Other causes of failure may be due to high insertion torque value of the implant exceeding $50 \mathrm{Ncm}$, which may have 
resulted in the transmission of high compressive stresses to the adjacent bone, in addition to compromising osseintegration success. This agrees with Sotto-Maior, Rocha, Almeida, Freitas-Júnior, Anchieta and Cury in (2010) (12) who stated that high insertion torques above $50 \mathrm{Ncm}$ can generate high compressive stresses to the peri-implant tissues therefore, causing deficient blood supply and bone ischemia during the osseointegration phase and early failure of the implant usually within the first month after placement.

Regarding our technique, it has many advantages as we place the implant guided by the retained roots, which act as a template for exact placement of the implant in an ideally prosthetic position. Furthermore, it prevents the slippage of the drills in the extraction sockets which is encountered in the traditional technique. Moreover, it avoids surgical complications of limited interocclusal distance in posterior segment as this problem is encountered when using surgical guides making insertion of the drills through the surgical guide impossible. In addition, this technique is cost effective compared to the computer guided implant placement technique. This agrees with the study done by Mahesh et al., in (2016) (13) and Joshi, Suhar, \& Gupta, in (2017) (14).

Some limitations of this technique are increased hardness of the root tissue, which may result in longer clinical time and greater risk of increasing bone temperature at the osteotomy site and of affecting the normal healing process because of the remains of dental tissue from drilling. Regarding the latter point, Davarpanah and Szmukler-Moncler in (2009) (15) made a study on five patients; according to the results, dental debris did not seem to adversely affect implant osseointegration, but there was little scientific evidence on this latter point, so caution is recommended, with an emphasis on copious irrigation and meticulous surgical debridement (16).

In our study, accuracy of implant placement was evaluated by measuring the overall deviations between virtually planned and surgically placed dental implants based on the superimposition of the preoperative and postoperative CBCT images.

Results showed that implants placed with the tooth guided technique had a range of horizontal deviation at the coronal side of the implant of $0.20-1.90$ with an average of $0.99 \pm$ $0.51 \mathrm{~mm}$.

Horizontal deviations at the apex of the implants had a range of $0.7-2.3 \mathrm{~mm}$ with an average of $1.28 \pm 0.50 \mathrm{~mm}$. Angular deviations between the long axis of the placed implants to the virtual implants had a range of $0.51-9.23$ degrees with an average of $3.78 \pm 3.22$ degrees.

The results of this technique showed ideal implant position compared to the traditional technique and this agrees with Scarano in (2017) (6).

In comparison to the accuracy of immediate implants placed using static surgical guides, both techniques showed an ideal prosthetic placed implants, and this was in agreement with Di Giacomo et al., in (2005) (17) and Joshi et al., in (2017) (14).

In our technique, accuracy was attributed to precise positioning and angulation of the implant bed preparation in the presence of any inter-radicular bone septa at multi-rooted extraction sockets. In addition, it should be emphasized that when this technique is applied, the initially retained remaining roots serve as an ideal template for the emergence profile of the tooth to be replaced. This was in agreement with Rohra et al., in (2017) (18).

Regarding implant stability, each implant was assessed by osstell device. In this study, the mean implant stability immediately postoperative was (55.89 \pm 15.53) indicating high primary stability, while the mean implant stability after 3 months increased to $(66.33 \pm 17.03)$.

These readings of high stability were attributed to the interradicular bone that could be routinely preserved in the lateral aspects of the implant drill hole, so the bone apical to the fundus of the socket may not have been the only factor that contributed to implant stability. This agrees with Rebele et al., in (2013) (19) who stated an increased stability following tooth guided immediate implant placement. Also this agrees with Scarano in (2017) (6) who stated that all implants placed with this technique had higher stability compared to the traditional technique of bed preparation after tooth removal.

Regarding bone density, it increased gradually immediately after implant placement till 6 month postoperative. This increase was due to healing ability of the bone and formation of new bone in the extraction sockets and around the implants. The increase in peri-implant bone density starting from the first month to the end of the 6 months of the evaluation period indicates osseointegration of all implants. That was in agreement with Albrektsson, Jansson, and Lekholm, in (1986) (20) and Parithimarkalaignan, and Padmanabhan, in (2013) (21).

Atraumatic extraction technique is a perquisite for successful immediate implant placement as it aid in maintaining the maximum amount of bone engaging the implant $(22,23)$. Atraumatic extraction will allow for the preservation of buccal plate of bone as well as inter-radicular bone, preventing their perforations or fracture without which an immediate implant placement might be contraindicated. This was in agreement with Acocella, Bertolai, \& Sacco, in (2010) (24) and Douglass and Merin in (2002) (22) who stated that atraumatic extraction and preservation of bone is mandatory for implant stability and success.

In our study, bone graft was not placed in the extraction sockets after implant placement and the sockets were left to heal by secondary intention, although the gap distance was $>2$ $\mathrm{mm}$. This was in accordance with Tarnow and Chu in (2011) (25) who stated that osseointegration occurs with excessive horizontal gap distance of an implant placed into an immediate extraction socket without primary flap closure, a bone graft, or a barrier membrane, this was also in agreement with Schwartz-Arad \& Chaushu (26) who stated that there is no consensus regarding the need for gap filling.

In our study, Ideal three dimensional implant placement guided by the roots of the tooth was significant in successful outcome of the final prosthesis. The final prosthesis was in an ideal prosthetic position, which led to even distribution of force on the implant, perfect emergence profile of the prosthesis and good plaque control by the patients. This was in agreement with Scarano in (2017) (6).

\section{CONCLUSION}

From the results of this study it was concluded that tooth guided immediate implant placement at molar region is a novel technique for easy and safe implant insertion at molar sites. 


\section{CONFLICT OF INTEREST}

The authors declare that they have no conflicts of interest.

\section{REFERENCES}

1. Schulte W, Heimke G. The Tubinger immediate implant. Die Quintessenz. 1976;27:17-23.

2. Botticelli D, Renzi A, Lindhe J, Berglundh T.Implants in fresh extraction sockets: A prospective5-year follow-up clinical study. Clin Oral Implants Res. 2008;19:1226-32.

3. Schwartz-Arad D, Laviv A, Levin L. Survival of immediately provisionalized dental implants placed immediately into fresh extraction sockets. J Periodontol. 2007;78:219-23.

4. Chen ST, Wilson TG, Jr., Hammerle CH. Immediate or early placement of implants following tooth extraction: review of biologic basis, clinical procedures, and outcomes. Int J Oral Maxillofac Implants. 2004;19 Suppl:12-25.

5. Moy PK, Nishimura GH, Pozzi A, Danda AK. Single implants indorsalareas -Asystematic review. Eur J Oral Implantol. 2016;9(Suppl 1):S163-72.

6. Scarano A. Traditional postextractive implant site preparation compared with pre-extractive interradicular implant bed preparation in the mandibular molar region, using an ultrasonic device: A randomized pilot study. Int J Oral Maxillofac Implants. 2017;32:655-60.

7. Rodriguez-Tizcareño MH, Bravo-Elores C. Anatomically Guided Implant Site Preparation Technique at Molar Sites. Implant Dent. 2009;18:393-401.

8. Barone A, Toti P, Quaranta A, Derchi G, Covani U. The Clinical Outcomes of Immediate Versus Delayed Restoration Procedures on Immediate Implants: A Comparative Cohort Study for Single-Tooth Replacement. Clin Implant Dent Relat Res. 2015;17:1114-26.

9. Atieh MA, Payne AG, Duncan WJ, de Silva RK, Cullinan MP. Immediate placement or immediate restoration/loading of single implants for molar tooth replacement: a systematic review and meta-analysis. Int J Oral Maxillofac Implants. 2010;25:401-15.

10. Peñarrocha M, Uribe R, Balaguer J. Immediate implants after extraction. A review of the current situation. Med Oral. 2004;9:234-42.

11. Tolstunov L. Dental implant success-failure analysis: concept of implant vulnerability. Implant Dent. 2006;15:341-6.

12. Sotto-Maior BS, Rocha EP, de Almeida EO, Freitas-Júnior AC, Anchieta RB, Del Bel Cury AA. Influence of high insertion torque on implant placement: an anisotropic bone stress analysis. Braz Dent J. 2010;21:508-14.
13. Mahesh L, Kurtzman GM, Schwartz D, Shukla S. Residual roots as an anatomical guide for implant placement: case series with two-year follow-up. J Oral Implantol. 2016;42:285-8.

14. Joshi V, Suhag V, Gupta S. Multirooted tooth guided immediate implant placement in molar sites: A report of two cases. Int J Curr Res. 2017;9:44967-9.

15. Davarpanah M, Szmukler-Moncler S. Unconventional implant treatment: I. Implant placement in contact with ankylosed root fragments. A series of five case reports. Clin Oral Implants Res. 2009;20:851-6.

16. Valenzuela S, Olivares JM, Weiss N, Benadof D. Immediate implant placement by interradicular bone drilling before molar extraction: clinical case report with one-year follow-up. Case Rep Dent. 2018;2018:6412826.

17. Di Giacomo GA, Cury PR, de Araujo NS, Sendyk WR, Sendyk CL. Clinical application of stereolithographic surgical guides for implant placement: preliminary results. J Periodontol. 2005;76:503-7.

18. Rohra E, Mistry G, Joshi T, Khanvilkar U. Implant bed preparation for immediate implantation in molar region: An alternative approach. JDMS. 2017;16:48-50.

19. Rebele SF, Zuhr O, Hürzeler MB. Pre-extractive interradicular implant bed preparation: case presentations of a novel approach to immediate implant placement at multirooted molar sites. Int $\mathrm{J}$ Periodontics Restorative Dent. 2013;33:89-96.

20. Albrektsson T, Jansson T, Lekholm U. Osseointegrated dental implants. Dent Clin North Am. 1986;30:151-74.

21. Parithimarkalaignan S, Padmanabhan TV. Osseointegration: an update. J Indian Prosthodont Soc. 2013;13:2-6.

22. Douglass GL, Merin RL. The immediate dental implant. J Calif Dent Assoc. 2002;30:362-5,368-74.

23. Wagenberg BD, Ginsburg TR. Immediate implant placement on removal of the natural tooth: retrospective analysis of 1,081 implants. Compend Contin Educ Dent. 2001,22:399-404, 406, 408 passim; quiz 412.

24. Acocella A, Bertolai R, Sacco R. Modified insertion technique for immediate implant placement into fresh extraction socket in the first maxillary molar sites: a 3-year prospective study. Implant Dent. 2010;19:220-8.

25. Tarnow DP, Chu SJ. Human histologic verification of osseointegration of an immediate implant placed into a fresh extraction socket with excessive gap distance without primary flap closure, graft, or membrane: a case report. Int J Periodontics Restorative Dent. 2011;31:515-21.

26. Schwartz-Arad D, Chaushu G. The ways and wherefores of immediate placement of implants into fresh extraction sites: a literature review. Journal of periodontology. 1997 Oct 1;68(10):915-23. 